\title{
Evaluation of inflammatory and metabolic impairments regarding depression dimensions: a case control study
}

Youmna Sabri ${ }^{* *}$ and Noha Abdelsalam²

\begin{abstract}
Background: Patients with depression are at a higher risk of developing serious medical problems such as diabetes mellitus, hypertension, and cardiovascular diseases, increasing the burden of depression and worsening its outcome.

Recent studies revealed the presence of low-grade systemic inflammation and metabolic impairments which are playing an important role in the development of these medical problems among patients with depression.

It should be noted that not all patients with depressive disorder have these immune or metabolic impairments.

The study aims to evaluate inflammatory and metabolic impairments among patients with depression through categorizing patients with depression into four dimensional profilers which are (1) atypical energy-related symptom dimension, (2) melancholic dimension, (3) childhood trauma dimension, and (4) anxious distress dimension.

Also, the current study will evaluate inflammatory and metabolic impairments among patients with depression and a control group.

Results: This study highlights that of the patients with metabolic impairments (including hypertension, impaired glycemic control, low/high-density lipoprotein, elevated triglycerides, and central obesity), all of them had reported significantly higher scores in the atypical, energy-related symptom dimension.

Also, patients with impaired glycemic control had reported significantly $(p<0.001)$ high scores in the anxious distress symptom dimension.

While patients with inflammatory impairment (serum CRP $>3 \mathrm{mg} / \mathrm{L}$ ) had significantly $(p=0.009$ ) reported higher scores in the childhood trauma symptom dimension.

In addition, statistically significant metabolic and inflammatory impairments are detected among the depression cases group in comparison with the control group.

Conclusions: This study found that patients with depression presented by atypical, energy-related symptoms were at a higher risk of metabolic impairments than other depression profilers.

Also, patients of the Anxious distress symptom dimension reported significant impaired glycemic control.

In addition, patients with depression of the childhood trauma dimension were associated with high levels of inflammatory marker (C-reactive protein).
\end{abstract}

\footnotetext{
*Correspondence: youmnasabri@yahoo.com

1 Department of Psychiatry, Faculty of Medicine, Mansoura University,

Mansoura, Egypt

Full list of author information is available at the end of the article
} 
Potential implications: These results can be applied clinically to improve treatment and prognosis in patients with depression. For example, depressed patients with atypical, energy-related symptoms should increase their daily physical activities and exercise, and they should follow a special diet. Also, anti-inflammatory medications could be added to depressed patients of the childhood trauma dimension.

Keywords: Depression profilers, Inflammatory, Metabolic impairment

\section{Background}

Major depressive disorder (MDD) is an important cause of disability in the 21st century [1] either in developed and developing countries.

The high disability of depression may be due to the relatively early age of its onset besides its chronic course [2].

In addition to the disabling symptoms of major depressive disorder, depressed patients are at a higher risk of comorbidity of serious medical problems such as diabetes mellitus, hypertension, and cardiovascular diseases, increasing the burden of depression and worsening its outcome [3].

Certainly, each of these disorders represents a real burden on general health, their comorbidity is a serious challenge. Understanding the pathogenesis of this comorbidity may help to face this challenge [4].

In addition to the genetic, biological, and psychosocial models involved in the pathogenesis of depression, recent studies revealed that the metabolic and immune-inflammatory impairments as higher levels of inflammatory markers, dyslipidemia, and metabolic syndrome are also reported [5]. Such findings represent serious risk factors for diabetes and cardiovascular diseases [6]. Also, the role of antidepressants cannot be ignored as certain antidepressants may predispose to cardio-metabolic dysfunction [7].

These comorbidities can be explained by the presence of shared pathogenesis linking these disorders such as altered circadian rhythms, abnormal hypothalamic-pituitary-adrenal axis (HPA axis), imbalanced neurotransmitters, and abnormal inflammatory process [8].

Low-grade inflammation is involved in the pathogenesis of depression and is increased in cardiovascular diseases [9]. Recent studies found that patients with depression compared with the control group have higher levels of inflammatory markers as C-reactive protein (CRP) and interleukin [10].

It should be noted that not all patients with depressive disorder have these immune or metabolic impairments. Latest studies revealed that only certain subgroups of patients with depression have these findings [11].

Recent studies about depression dimensions and subtypes are directing towards the dimensional approach of diagnosis and classification where the need for different dimensional regard is increasing [12].
Patients with depression who have an atypical symptom profile (increased appetite, weight gain, hypersomnia, fatigue, and lack of energy) are more likely to have impaired inflammatory and cardio-metabolic markers [13].

A new dimensional symptom profile known as "immuno-metabolic depression" [14].

However, updated research reported that both melancholic symptoms of depression [15] and depression with a comorbid anxiety disorder or with substantial anxiety features (defined by the DSM- 5 anxious distress specifier) [16] are associated with increased levels of inflammatory markers. Recently, anxiety disorders have been related to the metabolic syndrome [17].

Moreover, research on childhood trauma showed that patients with depression with childhood trauma had higher levels of inflammatory markers [18].

All these previous findings draw attention to create four-dimensional depression profilers: (1) atypical energy-related symptom dimension, (2) melancholic dimension, (3) childhood trauma dimension, and (4) anxious distress dimension. All four-dimensional depression profilers were standardized [19].

- The atypical, energy-related symptom dimension included the following items: increased appetite, increased weight, hypersomnia, leaden paralysis, and low energy.

- The melancholic symptom dimension included the following items: diurnal variation (mood worse in the morning), early morning awakening, and distinct quality of mood, inappropriate guilt, lack of appetite, loss of weight, and psychomotor agitation or retardation.

- The anxious distress symptom dimension included the following items: feeling tense, restlessness, concentration/worrying, fear of awful events, and feeling like losing control.

- The childhood trauma symptom dimension included the following items: emotional neglect, psychological abuse, physical abuse, and sexual abuse.

The comorbidity between depression and metabolic and immune-inflammatory impairments becomes an attractive area of research in the last years trying to 
highlight the need for following up metabolic and inflammatory changes of depression patients to prevent serious complications [20].

The aim of the present study was to evaluate inflammatory and metabolic impairments among patients with depression. This study was conducted by categorizing patients with depression into four-dimensional profilers which are (1)atypical energy-related symptom dimension, (2) melancholic dimension, (3) childhood trauma dimension, and (4) anxious distress dimension.

Laboratory assessment was done for evaluation of inflammatory and metabolic impairments among fourdimensional depression profilers and control group.

That may highlight the need for following up metabolic and inflammatory changes in patients with depression to prevent complication.

\section{Methods}

This is a cross-sectional study which was done in the Psychiatry Department, Faculty of Medicine, Mansoura University Hospitals, during the period from 1 May 2020 till 30 October 2020. Patients and control groups were selected for participation in this study according to the specified inclusion and exclusion criteria

\section{Patient group}

Inclusion criteria were as follow:

- Both sexes

- Age 20-40 (may be less affected by chronic physical illness or lifestyle factors)

- Diagnosed according to the diagnostic criteria of Diagnostic and Statistical Manual of Mental Disorders, Fourth Edition (DSM-IV) in a structured clinical interview as having major depressive disorder with or without comorbid anxiety

- Detecting the presence of depressive symptoms by having at least scoring (8-13) in the Hamilton Depression Rating Scale (HDRS). Detecting the presence of anxiety disorder by having at least scoring (< 17) in The Hamilton Anxiety Rating Scale (HAM-A)

- Under treatment with antidepressants only, or anidepressants with typical antipsycotics or atypical antipsychotics (ziprasdone, aripiprazole, quetiapine) as they are not affecting the metabolic profile markedly

Exclusion criteria were as follow:

- More than 40 years old

- Patients with other types of depression

- Patients with psychotic symptoms

- Patients with bipolar disorder

- Patients with other psychiatric disorders
- Patients under treatment with antipsychotics (risperidone, olanzpine, clozapine) as augmenting therapy (those types of antipsychotics are known to affect markedly the metabolic profile)

- Participants with $C R P>10 \mathrm{mg} / \mathrm{L}(n=2)$ were excluded due to the risk of acute infection or an active autoimmune disorder.

\section{Control group}

Inclusion criteria were as follow:

- Both sexes

- Age 20-40 (maybe less affected by chronic physical illness or lifestyle factors)

- The absence of depressive symptoms by having scored less than 8 in the Hamilton Depression Rating Scale (HDRS) and/or anxiety disorders by having less than 17 in The Hamilton Anxiety Rating Scale (HAM-A).

Exclusion criteria were as follow:

- More than 40 years old

- Patients with previous psychiatric history

- Participants with $C R P>10 \mathrm{mg} / \mathrm{L}(n=4)$ were excluded due to the risk of acute infection or an active autoimmune disorder.

\section{Sample size calculation}

Sample size calculation was based on the result of internal pilot study that is carried out on 20 cases and 20 matched control groups to estimate the sample size and revealed that the difference of metabolic impairments between groups is $20 \%$. Using G power program version 3.1.9.4 to calculate the sample size based on the expected difference of $20 \%$, using a 2-tailed test, $\alpha$ error $=0.05$ and power $=80.0 \%$, the total calculated sample size will be 80 in each group.

Finally, eighty $(n=80)$ patients and eighty $(n=80)$ controls participated in this study. All participants signed written informed consent and were informed that all information obtained will be confidential.

All included groups were subjected to a semi-structured interview to collect the following data including sociodemographic information (age, gender, residence, the level of education, and smoking).

\section{The psychometric assessment}

The assessment was done by the following: 
a. Hamilton Depression Rating Scale (HDRS) [21]: Arabic version translated by Lotfy Fateem [22] was applied to both groups (patients and control). HDRS is a multiple-item questionnaire used to indicate depression and as a guide to evaluating recovery. A total score of 8-13 is considered mild, 14-18 moderate depression, 19-22 severe depression, and $\geq 23$ very severe depression

b. The Hamilton Anxiety Rating Scale (HAM-A) [23]: The Arabic version prepared by Lotfy Fateem [24] was applied to both groups (patients and control). HAM-A is a questionnaire used to rate the severity of a patient's anxiety. Scoring a total of $<17$ is considered mild, 18-25 mild to moderate, and 26-30 moderate to severe.

c. Major depressive episodes were assessed with the Arabic version [25] of the Structured Clinical Interview for DSM- IV (SCID-1) [26, 27].

The patient group was divided into four-dimensional depression profilers based on a previous study done by Lamers et al. [19]; all four-dimensional depression profilers were standardized [19]. This classification was justified in their study by recent studies about depression dimensions, and subtypes are directing towards the dimensional approach of diagnosis and classification where the need for different dimensional regard is increasing [12]. A new dimensional symptom profile known as "immuno-metabolic depression" [14].

However, updated research reported that both melancholic symptoms of depression [15] and depression with a comorbid anxiety disorder are associated with increased levels of inflammatory markers. Recently, anxiety disorders have been related to metabolic syndrome [17].

Moreover, research on childhood trauma showed that patients with depression with childhood trauma had higher levels of inflammatory markers [18].

The items used in each dimensional depression profilers are from the 30-item version of the Inventory of Depressive Symptomatology (IDS) [28].

For preparing an Arabic version translation, back translation and validity were done in the Psychiatry Department, Faculty of Medicine, Mansoura University Hospitals (MUH) through the cooperation with a postgraduate member in the English language Department, Faculty of Arts, Mansoura University. The reliability was examined by test-retest method (with a 2 -week interval) with alpha cronbach coefficiency (0.87) through the cooperation with the Department of Community, Faculty of Medicine, Mansoura University.
The four-dimensional depression profilers

The following are the depression profilers:

- The atypical, energy-related symptom dimension consists of 5 items specific to immuno-metabolic depression (IMD) [6]. It included the following items: increased appetite, increased weight, hypersomnia, leaden paralysis, and low energy and ranges from 0 to 15 .

- The melancholic symptom dimension: all 8 melancholic features in the IDS are summed [29]: diurnal variation (mood worse in the morning), early morning awakening and distinct quality of mood, inappropriate guilt, lack of appetite, loss of weight, psychomotor agitation, or retardation. A score is ranging from 0 to 24 .

- The anxious distress symptom dimension: the dimension was determined by using three items from the IDS and two items of the Beck Anxiety Inventory(BAI) [30] that matched with the five criteria for the DSM-5 anxious distress specifier. The sum of these 5 items (IDS items feeling tense, restlessness, concentration/ worrying, and BAI items fear of awful events, feeling like losing control) resulted in a score ranging from 0 to 15 .

- The childhood trauma symptom dimension was done using the Childhood trauma interview (CTI) [31] assessing the occurrence and frequency of four types of abuse before age 16: (emotional neglect, psychological abuse, physical abuse, and sexual abuse) on a scale from 0 to 8 .

\section{Laboratory assessment of Inflammatory and metabolic markers}

This was done to both groups (patients and control):

- Inflammatory markers were determined from fasting morning blood plasma levels of CRP and measured by enzyme-linked immunosorbent assay (ELISA).

- We defined evidence of low-grade inflammation as serum $C R P>3 \mathrm{mg} / \mathrm{L}$, and no inflammation as $C R P<$ $3 \mathrm{mg} / \mathrm{L}$ [11].

- Cardio-metabolic dysregulation was defined using the factors of the metabolic syndrome: elevated blood pressure $(B P>130 / 85 \mathrm{mmHg}$ or use of antihypertensive medication), impaired glycemic control (fasting blood glucose concentration more than $5.6 \mathrm{mmol} / \mathrm{L}$ (38 $\mathrm{mmol} / \mathrm{mol}$ ) or diagnosed type 2 diabetes), low/ high-density lipoprotein cholesterol $(<1.03 \mathrm{mmol} / \mathrm{L}$ in male and $<1.30 \mathrm{mmol} / \mathrm{L}$ in female), elevated tri- 
glycerides (> $1.7 \mathrm{mmol} / \mathrm{L}$ ), and central obesity (waist circumference $\geq 102 \mathrm{~cm}$ (male), $\geq 88 \mathrm{~cm}$ ( female).

- All measures of assessment of inflammatory and metabolic impairments were done in the Outpatient Clinic of Internal Medicine, Faculty of Medicine, Mansoura University Hospitals (MUH).

- Waist circumference was measured with a measuring tape at the central point between the lowest front rib and the highest front point of the pelvis on light clothing.

- HDL cholesterol, triglycerides, and glucose were determined using routine standardized laboratory methods. Fasting plasma glucose samples were taken at 9 o'clock after $10 \mathrm{~h}$ of fasting.

\section{Statistical analysis and data interpretation}

Data were fed to the computer and analyzed using IBM SPSS Corp. Released 2013. IBM SPSS Statistics for Windows, Version 22.0. Armonk, NY: IBM Corp. Qualitative data were described using numbers and percentages. Quantitative data were described using the median (minimum and maximum) for nonparametric data and mean and the standard deviation for parametric data after testing normality using the Kolmogorov-Smirnov test. The significance of the obtained results was judged at the 0.05 level.

\section{Data analysis}

Nonparametric tests used were Mann-Whitney U \& Kruskal-Wallis tests to compare nonparametric variables. Linear regression analysis was used for the prediction of independent variables of continuous parametric outcome (depression scores after log transformation). Significant predictors in the correlation were entered into the regression model with the calculation of $R^{2}$ that quantities the effect of combined variables on the desired outcome and the prediction equation $(Y=\beta+a \times x)$

\section{Results}

Sociodemographic characteristics of the studied groups are demonstrated in Table 1.

Concerning the cases group, mean of age \pm SD was $(35.88 \pm 4.71), 50(62.5 \%)$ male, $30(37.5 \%)$ female, 48 $(60 \%)$ urban, and $32(40 \%)$ rural.

Thirteen $(16.2 \%)$ of the cases had primary education, $46(57.5 \%)$ had intermediate education while $21(26.2 \%)$ had high education.

Twenty four (30\%) of the cases were never smokers, 14 (17.5\%) were former smokers, and $42(52.5 \%)$ are current smokers which was statistically significant $(p=0.004)$.
Table 1 Sociodemographic characteristics of the studied groups

\begin{tabular}{|c|c|c|c|}
\hline & $\begin{array}{l}\text { Cases group } \\
N=80\end{array}$ & $\begin{array}{l}\text { Control group } \\
N=80\end{array}$ & Test of significance \\
\hline Age/years & & & $t=1.44$ \\
\hline Mean \pm SD & $35.88 \pm 4.71$ & $34.78 \pm 4.93$ & $p=0.151$ \\
\hline \multicolumn{4}{|l|}{ Sex } \\
\hline Male & $50(62.5 \%)$ & $52(65 \%)$ & $x^{2}=0.108$ \\
\hline Female & $30(37.5 \%)$ & $28(35 \%)$ & $p=0.74$ \\
\hline \multicolumn{4}{|l|}{ Residence } \\
\hline Urban & $48(60 \%)$ & $51(63.7 \%)$ & $x^{2}=0.0$ \\
\hline Rural & $32(40 \%)$ & $29(36.2 \%)$ & $p=1.0$ \\
\hline \multicolumn{4}{|l|}{ Education } \\
\hline Primary & $13(16.2 \%)$ & $9(11.2 \%)$ & $x^{2}=1.160$ \\
\hline Intermediate & $46(57.5 \%)$ & $53(77.5 \%)$ & $p=0.281$ \\
\hline High & $21(26.2 \%)$ & $18(22.5 \%)$ & \\
\hline \multicolumn{4}{|l|}{ Smoking } \\
\hline Never & $24(30 \%)$ & $44(55 \%)$ & $x^{2}=11.08$ \\
\hline Former & $14(17.5 \%)$ & $6(7.5 \%)$ & $p=0.004^{*}$ \\
\hline Current & $42(52.5 \%)$ & $30(37.5 \%)$ & \\
\hline
\end{tabular}

$t$ Student $t$ test; *statistically significant if $p<0.05 ; x^{2}$ chi-squared test

While in the control group, mean of age \pm SD was (34.78 \pm 4.93$)$, 52 (65\%) male, 28 (35\%) female, 51 (63.7\%) were urban while 29 (36.2\%) were rural.

Nine $(11.2 \%)$ of the control group had primary education, $53(77.5 \%)$ had intermediate education while 18 (22.5\%) had high education.

Forty four (55\%) of the control group were never smokers, 6(7.5\%) were former smokers, and 30 (37.5\%) are current smokers.

Table 2 shows metabolic and inflammatory impairments among the studied groups.

Statistically significant metabolic impairments (including hypertension, impaired glycemic control, low/highdensity lipoprotein, elevated triglycerides, and central obesity) and inflammatory impairment (serum CRP $>3$ $\mathrm{mg} / \mathrm{L}$ ) were detected among the cases group in comparison with the control group.

Table 3 shows depression dimensional profilers' score distribution (cases group).

- Atypical, energy-related symptom dimension: median (11.8), minimum (3), maximum (15)

- Melancholic symptom dimension: median (12), minimum (3), maximum (21)

- Childhood trauma index dimension: median (4), minimum (2), maximum (8)

- Anxious distress symptom dimension: median (3), minimum (3), maximum (15) 
Table 2 Metabolic and inflammatory impairments among cases and control groups

\begin{tabular}{llllll}
\hline & $\begin{array}{l}\text { Cases group } \\
\boldsymbol{n = 8 0}\end{array}$ & Percentage & $\begin{array}{l}\text { Control group } \\
\boldsymbol{n}=\mathbf{8 0}\end{array}$ & Percentage & Test of sig. \\
\hline Hypertension & 41 & 51.2 & 20 & 25 & $0.000^{*}$ \\
Impaired glycemic control & 36 & 45 & 18 & 22.5 & $0.003^{*}$ \\
Low/high-density lipoprotein & 64 & 80 & 43 & 53.8 & $<0.00^{*}$ \\
Elevated TGS & 61 & 75.3 & 31 & 38.8 & $<0.00^{*}$ \\
Central obesity & 57 & 41.2 & 38 & 47.5 & $0.001^{*}$ \\
Serum CRP $>3$ mg/L & 34 & 42.5 & 13 & 16.2 & $<0.02^{*}$ \\
\hline
\end{tabular}

*Statistically significant if $p<0.05$

Table 3 Depression dimensional profilers'score distribution among the cases group

\begin{tabular}{|c|c|c|c|c|}
\hline & $\begin{array}{l}\text { Atypical, energy-related } \\
\text { symptom dimension (0-15) }\end{array}$ & $\begin{array}{l}\text { Melancholic symptom } \\
\text { dimension }(0-24)\end{array}$ & $\begin{array}{l}\text { Childhood trauma index } \\
\text { dimension }(0-8)\end{array}$ & $\begin{array}{l}\text { Anxious distress } \\
\text { symptom dimension } \\
(0-15)\end{array}$ \\
\hline Mean & 12.48 & 11.2 & 3.6 & 5.8 \\
\hline Median & 11.8 & 12.0 & 4.0 & 3.0 \\
\hline Std. deviation & 7.66 & 6.52 & 2.34 & 5.81 \\
\hline Minimum & 3 & 3 & 2 & 3 \\
\hline Maximum & 15 & 21 & 8 & 15 \\
\hline
\end{tabular}

Table 4 The relations between depression dimensional profilers'scores and metabolic-inflammatory impairments

\begin{tabular}{|c|c|c|c|c|c|}
\hline & $\begin{array}{l}\text { Atypical, energy- } \\
\text { related symptom } \\
\text { dimension (0-15) } \\
\text { Median scores }(\%)\end{array}$ & $\begin{array}{l}\text { Melancholic } \\
\text { symptom } \\
\text { dimension (0-24) } \\
\text { Median scores (\%) }\end{array}$ & $\begin{array}{l}\text { Childhood trauma } \\
\text { symptom dimension } \\
(0-8) \\
\text { Median scores }(\%)\end{array}$ & $\begin{array}{l}\text { Anxious distress } \\
\text { symptom dimension } \\
(0-15) \\
\text { Median scores }(\%)\end{array}$ & Test of significance \\
\hline $\begin{array}{l}\text { Hypertensive patients } \\
(n=41)\end{array}$ & $12.3(82 \%)$ & $9.2(38.3 \%)$ & $3.3(41.2 \%)$ & $8.1(54 \%)$ & $p<0.001^{*}$ \\
\hline $\begin{array}{l}\text { Impaired glycemic } \\
\text { control } \\
(n=36)\end{array}$ & $12.9(86 \%)$ & $7.4(30.8 \%)$ & $2.6(32.5 \%)$ & $11.7(78 \%)$ & $p<0.001^{*}$ \\
\hline $\begin{array}{l}\text { Elevated LDL/HDL ratio } \\
(n=64)\end{array}$ & $11.8(78.6 \%)$ & $10.1(42 \%)$ & $3.1(38.7 \%)$ & $6.8(45.3 \%)$ & $p=0.038^{*}$ \\
\hline $\begin{array}{l}\text { Elevated TGS } \\
(n=61)\end{array}$ & $12.1(80.6 \%)$ & $8.9(37 \%)$ & $3.7(46.2 \%)$ & $6.4(42.6 \%)$ & $p=0.026^{*}$ \\
\hline $\begin{array}{l}\text { Central obesity } \\
(n=57)\end{array}$ & $13(86.6 \%)$ & $9(37.5 \%)$ & $3(37.5 \%)$ & $6(40 \%)$ & $p=0.004^{*}$ \\
\hline $\begin{array}{l}\text { Elevated CRP } \\
(n=34)\end{array}$ & $9.2(61.3 \%)$ & $11.3(47 \%)$ & $6.8(85 \%)$ & $7.3(48.6 \%)$ & $p=.009^{*}$ \\
\hline
\end{tabular}

*Statistically significant if $p<0.05$

Table 4 shows the relationships between depression dimensional profilers' scores and metabolic-inflammatory impairments.

- All patients with metabolic impairments (including hypertension, impaired glycemic control, low/highdensity lipoprotein, elevated triglycerides, and cen- tral obesity) had reported significantly higher scores in the atypical, energy-related symptom dimension.

- Also, patients with impaired glycemic control had reported significantly high scores in the anxious distress symptom dimension.

- While patients with inflammatory impairment (serum $C R P>3 \mathrm{mg} / \mathrm{L}$ ) had significantly reported 
Table 5 Linear regression for prediction of Log atypical energyrelated symptoms dimension

\begin{tabular}{llll}
\hline & $B$ & $\boldsymbol{T}$ & $\boldsymbol{p}$ \\
\hline Hypertension & .614 & 7.53 & $<.001^{*}$ \\
Impaired glycemic control & .321 & 1.31 & $.02^{*}$ \\
Elevated LDL/HDL ratio & .728 & 5.23 & $<.001^{*}$ \\
Central obesity & $\mathbf{0 . 2 4 5}$ & $\mathbf{7 . 2 1}$ & $0.04^{*}$ \\
\hline
\end{tabular}

*Statistically significant if $p<0.05$

higher scores in the childhood trauma symptom dimension.

Table 5 presents the linear regression for prediction (Log atypical energy-related symptoms dimension). Patients with higher scores at the atypical, energy-related symptom dimension were significantly $(p<0.001)$ hypertensive; their glycemic control was significantly impaired $(p=0.02)$ and low/high-density lipoprotein significantly $(p<0.001)$ impaired, and they significantly $(p=0.04)$ had central obesity.

Table 6 shows the linear regression for prediction (Log childhood trauma symptom dimension); patients with higher scores in the childhood trauma symptom dimension had significantly $(p<0.001)$ elevated C-reactive protein.

Table 7 shows the linear regression for prediction (Log anxious distress symptom dimension); patients with high scores in the Anxious distress symptom dimension had reported significantly impaired glycemic control.

\section{Discussion}

The purpose of the present study was to evaluate inflammatory and metabolic impairments among patients with depression. This study was conducted by categorizing depression patients into four-dimensional profilers which are (1) atypical energy-related symptom dimension, (2) melancholic dimension, (3) childhood trauma dimension, and (4) anxious distress dimension.

Laboratory assessment was done for evaluation of inflammatory and metabolic impairments among the

Table 6 Linear regression for prediction of Log childhood trauma symptom dimension

\begin{tabular}{llll}
\hline & $B$ & $\boldsymbol{T}$ & $\boldsymbol{p}$ \\
\hline Hypertension & 7.108 & 8.768 & $p=1.0$ \\
Elevated LDL/HDL ratio & 2.280 & 3.928 & $p=0.15$ \\
Central obesity & 10.284 & 12.381 & $p=0.72$ \\
Elevated CRP & 1.592 & 13.240 & $<.001^{*}$ \\
\hline
\end{tabular}

*Statistically significant if $p<0.05$
Table 7 Linear regression for prediction of Log anxious distress symptom dimension

\begin{tabular}{llll}
\hline & $B$ & $\boldsymbol{T}$ & $\boldsymbol{p}$ \\
\hline Hypertension & -.194 & -1.664 & .101 \\
Impaired glycemic control & .311 & 3.155 & $.002^{*}$ \\
Elevated LDL/HDL ratio & .183 & 1.869 & .066 \\
Elevated TGS & .042 & .450 & .654 \\
\hline
\end{tabular}

*Statistically significant if $p<0.05$

four-dimensional depression profilers and the control group.

Results of the current study revealed that all patients with metabolic impairments (including hypertension, impaired glycemic control, low/high-density lipoprotein, elevated triglycerides, and central obesity) had reported significantly higher scores in the atypical, energy-related symptom dimension.

These results are indicating more metabolic dysregulation in patients with higher scores in the atypical, energy-related symptom dimension.

According to [19], the atypical, energy-related symptom dimension included the following items: increased appetite, increased weight, hypersomnia, leaden paralysis, and low energy.

A possible explanation for these significant findings in this dimension of depression may be that increased appetite, hypersomnia, leaden paralysis, and low energy present in the atypical depression may lead to higher fat and carbohydrate intake as well as decreased daily physical activity and weight gain leading to significant impairment in blood glucose and lipid levels.

In the same area of research, Alshehri et al. [2] reported the association between abdominal obesity and depression, Simmons et al. [7] revealed that appetite changes are related to metabolic impairments in the depression subgroups.

The results of the current study are agreeing with prior similar studies showing that metabolic dysregulation is strongly related to atypical energy-related symptom dimension [32, 33].

Also, the results of $[34,35]$ confirmed our results that depression with atypical features is associated with an increase in waist circumference and fasting glucose and a higher incidence of metabolic impairment.

On the other hand, according to the results of this study, the melancholic symptom dimension is less likely to be linked to inflammatory and metabolic impairments.

This may be due to the lower appetite present in melancholic depression which will lead to lower fat and carbohydrate intake, resulting in lowering blood glucose and 
lipid levels and less be linked to inflammatory and metabolic impairments.

Recent studies [36, 37] had the same findings, reporting that melancholic forms of depression are associated with a lower prevalence of metabolic syndrome.

Also, the results of the current research highlighted that patients with impaired glycemic control had reported significantly high scores in the anxious distress symptom dimension.

This important finding can be explained as anxiety is frequently associated with poor metabolic functions and serious medical problems. Anxiety is associated with increased levels of the stress hormone cortisol which block the effect of insulin from taking glucose from blood into cells all over the body causing high blood sugar and finally impaired glycemic control.

A recent study [17] reported the association between anxiety and metabolic syndrome emphasizing the role that cortisol plays in this relation.

Another important finding in this study is that patients with inflammatory impairment (serum $C R P>3 \mathrm{mg} / \mathrm{L}$ ) had reported significantly higher scores in the childhood trauma symptom dimension.

This can be explained as childhood trauma (emotional neglect, psychological abuse, physical abuse, and sexual abuse) usually leads to heightened stress response in the childhood period leading to impaired emotional regulation, sleep problems, and autoimmune diseases. Also, childhood exposure to trauma can trigger a systematic inflammatory response.

These data are consistent with some recent studies [38-40] who had reported that childhood trauma was found to be significantly associated with higher inflammatory markers among patients with depression.

The last finding is the presence of statistically significant metabolic impairments (including hypertension, impaired glycemic control, low/high-density lipoprotein, elevated triglycerides, and central obesity) and inflammatory impairment (serum $C R P>3 \mathrm{mg} / \mathrm{L}$ ) among the cases group in comparison with the control group.

Patients with depressive disorder are prone to metabolic and inflammatory impairment due to their poor health-related behaviors. In addition to the genetic, biological, and psychosocial models involved in the pathogenesis of depression, recent studies revealed that the metabolic and immune-inflammatory impairments as higher levels of inflammatory markers, dyslipidemia, and metabolic syndrome are also reported. Such findings represent serious risk factors for diabetes and cardiovascular diseases [6]. Also, the role of antidepressants cannot be ignored as certain antidepressants may predispose to cardio-metabolic dysfunction [7].
Finally, this study highlights that all patients with metabolic impairments (including hypertension, impaired glycemic control, low/high-density lipoprotein, elevated triglycerides, and central obesity) had reported significantly higher scores in the atypical, energy-related symptom dimension.

Also, patients with impaired glycemic control had reported significantly high scores in the anxious distress symptom dimension while patients with inflammatory impairment (serum $C R P>3 \mathrm{mg} / \mathrm{L}$ ) had reported significantly higher scores in the childhood trauma symptom dimension.

Based on these results, these results can be applied clinically to improve treatment and prognosis in patients with depression. For example, depressed patients with atypical, energy-related symptoms should increase their daily physical activities and exercise, and they should follow a special diet. Also, anti-inflammatory medications could be added to depressed patients of the childhood trauma symptom dimension.

Before we conclude, we would like to discuss the limitations of this study. A limited number of studies on the dimensions of depression are available. Depression subtypes are often overlapping which acquires further assessment of the correlations between profilers.

Research on larger samples is needed to confirm our findings.

In addition, the previous medical history of those patients and drug treatment they receive for these conditions should have been taken into consideration.

Also, in the coming research in this area, the role of pharmacotherapy for depression should be more adjusted as it may predispose to cardio-metabolic dysfunction.

Finally, lifestyle-related behavior is a risk factor, which needs further assessment.

\section{Conclusions}

This study found that patients with depression presented by atypical, energy-related symptoms were at a higher risk of metabolic impairments than other depression profilers.

Also, patients of the anxious distress symptom dimension reported significant impaired glycemic control.

In addition, depressed patients of the childhood trauma dimension were associated with high levels of inflammatory marker (C-reactive protein).

\section{Potential implications}

These results can be applied clinically to improve treatment and prognosis in patients with depression. For example, patients with depression with atypical, energyrelated symptoms should increase their daily physical activities and exercise, and they should follow a 
special diet. Also, anti-inflammatory medications could be added to patients with depression of the childhood trauma dimension.

Future studies are needed to confirm findings and to benefit from these symptoms to develop an ideal treatment plan.

\begin{abstract}
Abbreviations
MDD: Major depressive disorder; HPA axis: Hypothalamic-pituitary-adrenal axis; CRP: C-reactive protein; LDL: Low-density lipoprotein; HDL: High-density lipoprotein; HDRS: Hamilton Depression Rating Scale; HAM-A: The Hamilton Anxiety Rating Scale; DSM-IV: Diagnostic and Statistical Manual of Mental Disorders, Fourth Edition; IDS: Inventory of Depressive Symptomatology; BAI: Beck Anxiety Inventory; CTI: Childhood trauma interview.
\end{abstract}

\section{Acknowledgment}

The authors are appreciative to all the patients who participated in this study.

\section{Authors' contributions}

YS was responsible for preparing of the study design, conducting the psychiatric assessment, analysis and interpretation of data manuscript, drafting, and revising. NA was responsible for conducting medical assessment and manuscript drafting and revising. Both authors read the final manuscript and agreed on its contents. Both authors have read and approved the final manuscript.

\section{Funding}

No funds were received for this study.

\section{Availability of data and materials}

Data are available with the request from the corresponding author.

\section{Declarations}

\section{Ethics approval and consent to participate}

All subjects signed a written informed consent form before joining the study. This study was submitted to the Institutional Research Board (IRB), Faculty of Medicine, Mansoura University; reference code is "R.21.03.1282".

\section{Consent for publication}

Not applicable.

\section{Competing interests}

The author reported no financial interests or conflicts of interest.

\section{Author details}

${ }^{1}$ Department of Psychiatry, Faculty of Medicine, Mansoura University, Mansoura, Egypt. ${ }^{2}$ Internal Medicine Department, Faculty of Medicine, Mansoura University, Mansoura, Egypt.

Received: 2 December 2021 Accepted: 14 January 2022

Published online: 01 February 2022

\section{References}

1. Disease and Injury Incidence and Prevalence Collaborators (2017) Global, regional, and national incidence, prevalence, and years lived with disability for 354 diseases and injuries for 195 countries and territories, 1990-2017: a systematic analysis for the Global Burden of Disease Study. Lancet (London, England) 392:1789-1858 https://doi.org/10.1016/S01406736(18)32279-7

2. Alshehri T, Boonen S, de Mutsert R, Penninx B, Rosendaal F, le Cessie S (2019) The association between overall and abdominal adiposity and depressive mood: a cross-sectional analysis in 6459 participants. Psychneuroendocrinology 110:104429 https://doi.org/10.1016/j.psyneuen. 2019
3. Otte C, Gold SM, Penninx BW, Pariante CM, Etkin A, Fava M (2016) Major depressive disorder. Nat Rev Dis Prim 2:16065 https://doi.org/10.1038/ nrdp.2016.65

4. Perry BI, Olteana BP, Jones PB, Khandakera GM (2020) Cardio metabolic risk in young adults with depression and evidence of inflammation: a birth cohort study. Psychneuroendocrinology. 116:104682

5. Cao B, Chen Y, Brietzke E, Cha D, Shaukat A, Pan Z (2018) Leptin and adiponectin levels in major depressive disorder: a systematic review and meta-analysis. J Affect Disord. 238:101-110 https://doi.org/10.1016/j.jad. 2018.05.008

6. Milaneschi Y, Lamers F, Berk M, Penninx BWJH (2020) Depression heterogeneity and its biological underpinnings from atypical to immunometabolic depression. Biol Psychiatry. https://doi.org/10.1016/j.biopsych. 2020.01.014

7. Simmons WK, Burrows K, Avery JA, Kerr KL, Taylor A, Bodurka J (2018) Appetite changes reveal depression subgroups with distinct endocrine, metabolic, and immune states. Mol Psychiatry https://doi.org/10.1038/ s41380-018-0093-6

8. Khandaker GM, Zuber V, Rees JMB, Carvalho L, Mason AM, Foley CN (2019) Shared mechanisms between coronary heart disease and depression: findings from a large UK general population-based Cohort. Mol Psychiatry in press

9. Lee $\mathrm{CH}$, Giuliani F (2019) The role of inflammation in depression and fatigue. Front Immunol 10:1696

10. Leighton SP, Nerurkar L, Krishnadas R, Johnman C, Graham GJ, Cavanagh J (2018) Chemokines in depression in health and in inflammatory illness: a systematic review and meta-analysis. Mol Psychiatry 23:48-58

11. Osimo EF, Baxter LJ, Lewis G, Jones PB, Khandaker GM (2019) Prevalence of low-grade inflammation in depression: a systematic review and metaanalysis of CRP levels. Psychol Med https://doi.org/10.1017/S003329171 9001454

12. Glaus J, Von Känel R, Lasserre AM, Strippoli M-PF, Vandeleur CL, Castelao E et al (2018) Mood disorders and circulating levels of inflammatory markers in a longitudinal population-based study. Psychol Med 48:961-973 https://doi.org/10.1017/S0033291717002744

13. Perry BI, Khandaker GM, Marwaha S, Thompson A, Zammit S, Singh SP (2020) Insulin resistance and obesity, and their association with depression in relatively young people: findings from a large UK birth cohort. Psychol Med 50:556-565

14. Lamers F, Milaneschi Y, Smit JH, Schoevers RA, Wittenberg G, Penninx BWJH (2019) Longitudinal association between depression and inflammatory markers: results from the Netherlands study of depression and anxiety. Biol Psychiatry 85:829-837 https://doi.org/10.1016/j.biopsych. 2018.12.020

15. Yang C, Tiemessen KM, Bosker FJ, Wardenaar KJ, Lie J, Schoevers RA (2018) Interleukin, tumor necrosis factor-a and C-reactive protein profiles in melancholic and non-melancholic depression: a systematic review. J Psychosom Res 111:58-68 https://doi.org/10.1016/j.jpsychores.2018.05. 008

16. Gaspersz R, Lamers F, Wittenberg G, Beekman ATF, Van Hemert AM, Schoevers (2017) Brain Behav Immun 88:174-183

17. Tang F, Wang G, Lian Y (2017) Association between anxiety and metabolic syndrome: a systematic review and meta-analysis of epidemiological studies. Psychneuroendocrinology 77:112-121 https://doi.org/10.1016/j. psyneuen.2016.11.025

18. Suglia SF, Koenen KC, Boynton-Jarrett R, Chan PS, Clark CJ, Danese A (2018) Childhood and adolescent adversity and cardiometabolic outcomes: a scientific statement from the American heart association. Circulation 137 https://doi.org/10.1161/CIR.0000000000000536

19. Femke L, Milaneschia Y, Vinkersa CH, Schoeversc RA, Giltayd EJ (2020) Depression profilers and immune-metabolic dysregulation: Iongitudinal results from the NESDA study. Brain Behav Immun 88:174-183

20. Goldstein JM, Hale T, Foster SL, Tobet SA, Handa RJ (2019) Sex differences in major depression and comorbidity of cardiometabolic disorders: impact of prenatal stress and immune exposures. Neuropsychopharmacology 44:59-70

21. Hamilton M (1980) Rating. Depressive patients. J Clin Psychiatry 41:21-24 PMID 7440521

22. Fatim L (1994) Hamilton checklist of symptoms of depressive illness (Arabic version). Anglo Library, Cairo, Egypt 
23. Maier W, Buller R, Philipp M, Heuser I (1988) The Hamilton Anxiety Scale: reliability, validity and sensitivity to change in anxiety and depressive disorders. J Affect Disord. 14(1):61-68

24. Fatim $L$ (1994) Hamilton checklist of anxiety (Arabic version). Anglo Library, Cairo, Egypt

25. El Missiry A, Sorour A, Sadek A, Fahy T, Abdel Mawgoud M, Asaad T (2004) Homicide and psychiatric illness: an Egyptian study [MD thesis]. Faculty of Medicine, Ain Shams University, Cairo

26. American Psychiatric Association Diagnostic and statistical manual of mental disorders DSM-IV-TR, 2000. Text Revision, 4th edn. American Psychiatric Association

27. First MB, Spitzer RL, Gibbon M, Williams JBW, Benjamin LS (1997) Structured Clinical Interview for DSM-IV-clinician version (SCID-CV) (user's guide interview). American Psychiatric Press. G, Washington, DC

28. Rush AJ, Gullion CM, Basco MR, Jarrett RB, Trivedi MH (1996) The Inventory of Depressive Symptomatology (IDS): psychometric properties. Psychol Med 26:477-486

29. Khan AY, Carrithers J, Preskorn SH, Lear R, Wisniewski SR, Rush JA (2006) Clinical and demographic factors associated with DSM-IV melancholic depression. Ann Clin Psychiatry

30. Beck AT, Epstein N, Brown G, Steer RA (1988) An inventory for measuring clinical anxiety: psychometric properties. J Consult Clin Psychol 56:893-897

31. De Graaf R, Bijl RV, Have T, BeekmanA VWA, Spijker J (2004) Risk factors for 12-month rapid onset of comorbidity of common mental disorders: findings from the Netherlands Mental Health Survey and Incidence Study. Am J Psychiatry 109:55-63

32. Lamers F, Milaneschi Y, De Jonge P, Giltay EJ, Penninx BWJH (2018) Metabolic and inflammatory markers: associations with individual depressive symptoms

33. Norbert S, Deschênes SS, Burns RJ, Danna SM, Franco OH, Arfan Ikram M (2018) Cardio metabolic dysregulation and cognitive decline: potential role of depressive symptoms. Br J Psychiatry 212:96-102. https://doi.org/ 10.1192/bjp.2017.26

34. Lasserre AM, Strippoli M-PF, Glaus J, Gholam-Rezaee M, Vandeleur CL, Castelao E (2016) Prospective associations of depression subtypes with cardio-metabolic risk factors in the general population. Mol Psychiatry https://doi.org/10.1038/mp.2016.178

35. Polanka BM, Vrany EA, Patel J, Stewart JC (2017) Depressive disorder subtypes as predictors of incident obesity in US adults: moderation by race/ ethnicity. Am J Epidemiol:1-9 https://doi.org/10.1093/aje/kwx030

36. Vogelzangs N, Beekman ATF, Van Reedt Dortland AKB, Schoevers RA, Giltay EJ, De Jonge P (2014) Inflammatory and metabolic dysregulation and the 2-year course of depressive disorders in antidepressant users. Neuropsychopharmacology 39:1624-1634 https://doi.org/10.1038/npp. 2014.9

37. Silva D.A., Coutinho E. da S.F., Ferriani L.O., Viana M.C (2019). Depression subtypes and obesity in adults: a systematic review and meta-analysis. Obes Rev Orb. https://doi.org/10.1111/obr.12966. 12966.

38. De Punder K, Entringer S, Heim C, Deuter CE, Otte C, Wingenfeld K (2018) Inflammatory measures in depressed patients with and without a history of adverse childhood experiences. Front Psychiatry 9:610 https://doi.org/ 10.3389/fpsyt.2018.00610

39. Munjiza A, Kostic M, Pesic D, Gajic M, Markovic I, Tosevski DL (2018) Higher concentration of interleukin 6 - a possible link between major depressive disorder and childhood abuse. Psychiatry Res. 264:26-30 https://doi.org/10.1016/j.psychres.2018.03.072

40. Müller N, Krause D, Barth R, Myint AM, Weidinger E, Stettinger W (2019) Childhood adversity and current stress are related to pro- and antiinflammatory cytokines in major depression. J Affect Discord 253:270276 https://doi.org/10.1016/j.jad.2019.04.088

\section{Publisher's Note}

Springer Nature remains neutral with regard to jurisdictional claims in published maps and institutional affiliations.

\section{Submit your manuscript to a SpringerOpen ${ }^{\circ}$ journal and benefit from:}

- Convenient online submission

- Rigorous peer review

- Open access: articles freely available online

- High visibility within the field

- Retaining the copyright to your article

Submit your next manuscript at $\boldsymbol{\nabla}$ springeropen.com 